$$
\begin{aligned}
& \text { Pontifícia Universidade } \text { Católica }_{\text {a }} \\
& \text { DO RIO DE JANEIRO }
\end{aligned}
$$

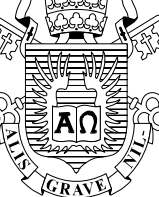

Felipe Ernesto Lamm Pereira

Determinação do Intervalo de Manutenção Programada da Proteção de Linhas de Transmissão Considerando-se Penalidades Associadas à Indisponibilidade

\title{
Tese de Doutorado
}

Tese apresentada ao Programa de Pós-graduação em Engenharia Elétrica do Departamento de Engenharia Elétrica da PUC-Rio como parte dos requisitos parciais para obtenção do título de Doutor em Engenharia Elétrica.

Orientador: Dr. Ricardo Bernardo Prada Co-Orientador: Dr. Albert Cordeiro Geber de Melo

Rio de Janeiro Outubro de 2008 


\title{
Pontifícia Universidade Católica $_{\text {a }}$ DO RIO DE JANEIRO \\ $\frac{\overline{A \Omega}}{\text { GRAVE }}$
}

\section{Felipe Ernesto Lamm Pereira}

Determinação do Intervalo de Manutenção Programada da Proteção de Linhas de Transmissão Considerando-se Penalidades Associadas à Indisponibilidade

Tese apresentada como requisito parcial para obtenção do grau de Doutor pelo Programa de Pós-Graduação em Engenharia Elétrica do Departamento de Engenharia Elétrica do Centro Técnico Científico da PUC-Rio. Aprovada pela Comissão Examinadora abaixo assinada.

\author{
Dr. Ricardo Bernardo Prada \\ Orientador \\ Departamento de Engenharia Elétrica/PUC-Rio \\ Dr. Albert Cordeiro G. de Melo \\ Co-Orientador \\ CEPEL \\ Dra. Maria da Guia da Silva \\ UFMA
}

Dra. Maria Elvira Pineiro Maceira

CEPEL

Dra. Paulo Roberto de Holanda Sales

ELETROBRÁS

Dr. Álvaro de Lima Veiga Filho Departamento de Engenharia Elétrica - PUC-Rio

Prof. José Eugenio Leal Coordenador Setorial do Centro Técnico Científico - PUC-Rio

Rio de Janeiro, 10 de Outubro de 2008 
Todos os direitos reservados. É proibida a reprodução total ou parcial do trabalho sem autorização da universidade, do autor e do orientador.

\section{Felipe Ernesto Lamm Pereira}

Graduado em Engenharia Elétrica na Pontifícia Universidade Católica do Rio de Janeiro em 2001. Mestre em Engenharia Elétrica pela Pontifícia Universidade Católica do Rio de Janeiro em 2003. Dedicado a tempo integral à pesquisa em Sistemas de Energia Elétrica na PUC-Rio.

Ficha Catalográfica

Pereira, Felipe Ernesto Lamm

Determinação do intervalo de manutenção programada da proteção de linhas de transmissão considerando-se penalidades associadas à indisponibilidade / Felipe Ernesto Lamm Pereira ; orientador: Ricardo Bernardo Prada ; co-orientador: Albert Cordeiro Geber de Melo. - 2008.

212 f. ; $30 \mathrm{~cm}$

Dissertação (Mestrado em Engenharia Elétrica) - Pontifícia Universidade Católica do Rio de Janeiro, Rio de Janeiro, 2008.

Inclui bibliografia

1. Engenharia Elétrica - Teses. 2. Confiabilidade. 3. Gerenciamento de ativos. 4. Manutenção centrada na confiabilidade. 5. Análise de risco. 6. Parcela variável. 7. Processos de Markov. I. Prada, Ricardo Bernardo. II. Melo, Albert Cordeiro Geber de. III. Pontifícia Universidade Católica do Rio de Janeiro. Departamento de Engenharia Elétrica. IV. Título. 
Aos meus pais Vera Ruth Lamm Pereira e Antonio Fernandes Pereira pelo amor, apoio e confiança. 


\section{Agradecimentos}

A Deus.

Ao meu irmão João Maurício Lamm Pereira por seu apoio e ajuda ao longo deste período.

A Vanda Maria de Sá, por seus bons conselhos, amor e por estar sempre a meu lado.

Muito especialmente, agradeço aos meus orientadores Ricardo Bernardo Prada e Albert Cordeiro Geber de Melo pelo permanente apoio nas diferentes etapas do desenvolvimento desta tese, por suas atenções e paciência nas discussões dos diferentes aspectos relacionados ao tema de pesquisa.

À CNPq, Nordeste Transmissora de Energia S/A, CEPEL e PUC-Rio, pelos auxílios concedidos, sem os quais este trabalho não poderia ter sido realizado.

Aos professores que participaram da comissão examinadora: Maria da Guia da Silva, Maria Elvira Pineiro Maceira, Paulo Roberto de Holanda Sales e Álvaro de Lima Veiga Filho, pela enorme contribuição dada a este trabalho e por suas sugestões para trabalhos futuros.

A todos os amigos do curso de Pós-Graduação em Engenharia Elétrica, e aos professores e demais funcionários do Departamento de Engenharia Elétrica da PUC-Rio. 


\section{Resumo}

Pereira, Felipe Ernesto Lamm; Prada, Ricardo Bernardo (Orientador). Determinação do Intervalo de Manutenção Programada da Proteção de Linhas de Transmissão Considerando-se Penalidades Associadas à Indisponibilidades. Rio de Janeiro, 2008. 212p. Tese de Doutorado - Departamento de Engenharia Elétrica, Pontifícia Universidade Católica do Rio de Janeiro.

Uma empresa de energia elétrica tem por obrigação garantir a continuidade e a qualidade do serviço prestado. A fim de incentivar a qualidade do serviço, a ANEEL introduziu penalidades nos contratos com as concessionárias de serviços públicos de transmissão de energia elétrica caso as instalações de transmissão sejam desligadas, por acidente, falha de equipamento ou manutenção programada. Com base nas práticas de Manutenção Centrada na Confiabilidade, este trabalho propõe uma determinação dos intervalos de manutenção programada para minimizar as penalidades pagas por uma empresa de transmissão de energia elétrica devido ao desligamento de equipamentos. Desenvolveu-se um método para a determinação das probabilidades em regime permanente dos estados que representam os desligamentos do sistema de proteção e suas respectivas taxas de transição, utilizando-se técnicas de Freqüência e Duração. A taxa de falha dos equipamentos foi determinada tendo como base dados do sistema de proteção coletados ao longo de onze anos, onde as manutenções programadas foram realizadas a cada três anos. Determinou-se a taxa de falha do sistema de proteção considerando-se outros intervalos fixos para a manutenção programada. Para cada intervalo fixo de manutenção, supôs-se que a taxa de falha encontrase na região onde ela é constante. Nesta região as falhas ocorrem aleatoriamente é podem ser descritas por uma função densidade de probabilidade exponencial. Para uma concessionária, é preferível assumir mais riscos a ter que pagar penalidades por manutenções programadas, enquanto que para uma outra é recomendável ser cautelosa é pagar mais por realização de manutenções programadas assumindo menos riscos de pagamentos por indisponibilidade não programada. Foram experimentados níveis de riscos aceitáveis ao problema utilizando-se técnicas da teoria econômica.

\section{Palavras-chave}

Confiabilidade; Gerenciamento de Ativos; Manutenção Centrada na Confiabilidade; Análise de Risco; Parcela Variável; Processos de Markov. 


\section{Abstract}

Pereira, Felipe Ernesto Lamm Pereira; Prada, Ricardo Bernardo (Advisor). Determining Maintenance Scheduling of Transmission Systems Considering Penalties Associated With Unavailability. Rio de Janeiro, 2008. 212p. Doctorate Thesis - Departamento de Engenharia Elétrica, Pontifícia Universidade Católica do Rio de Janeiro.

An electrical utility has for obligation to guarantee the continuity and the quality of the given service. In order to stimulate the quality of the service the Brazilian energy agency introduced penalties in contracts with the electrical utilities of transmission in case the transmission installations is off for accident, failure of equipment or scheduled maintenance. On the basis of practical Reliability Centered Maintenance, this thesis considers a determination of maintenance intervals to minimize the economical impacts due to equipment disconnection. These disconnections are represented through the probabilistic model of the protection of transmission systems. A method for determination of the steady states probabilities of the protection system and its transitions rates was developed using techniques of Frequency and Duration. The failure rate of the component was determined using data collected from protection of transmission systems throughout eleven years, where the maintenances had been carried through every three years. It was determined the protection failure rate considering others intervals of maintenance. For each interval of maintenance, one assumed that the failure rate meets the useful life period and is characterized by a constant hazard rate. The useful life period follows a good approximation to an exponential curve. For a utility it is preferable to take more risks of component failure than to have to pay penalties for maintenances, whereas for another one is recommendable to be cautious and pay more for accomplishment of maintenances taking little risks of payments for unavailability do to component failure. Techniques of the economic theory had been tried in order to determine reasonable levels of acceptable risks to the problem.

\section{Keywords}

Reliability; Asset Management; Reliability Centered Maintenance; Markov Process; Risk; Penalties Associated With Component Unavailability. 


\section{Sumário}

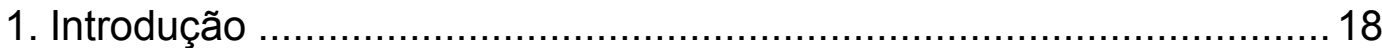

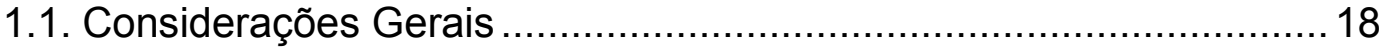

1.2. Impacto da Indisponibilidade de Equipamentos na Receita de Agentes

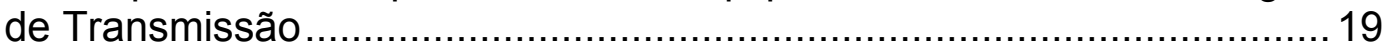

1.2.1. Fundamentos Contratuais ..................................................... 22

1.2.2. Conceitos Básicos da Resolução ANEEL 270/07 ......................... 22

1.2.3. Desligamentos Programados ................................................ 23

1.2.4. Desligamentos Não Programados ............................................ 24

1.2.5. Contabilização das Indisponibilidades .......................................... 24

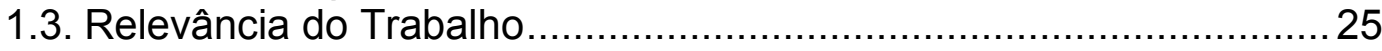

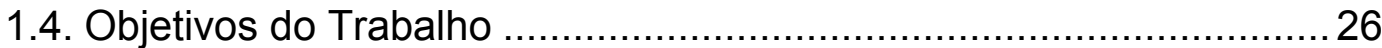

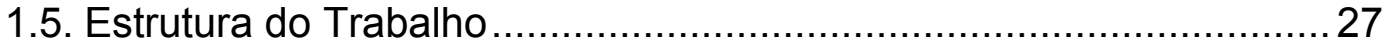

2. Gerenciamento de Ativos em Sistemas de Energia Elétrica ................29

2.1. Gerenciamento de Ativos: Visão Geral .......................................... 29

2.1.1. Filosofia Empresarial ......................................................... 30

2.1.2. Estrutura Organizacional ..................................................... 30

2.1.3. Planejamento com Menor Risco .............................................. 32

2.2. Gerenciamento de Ativos Aplicado em Sistemas de Energia

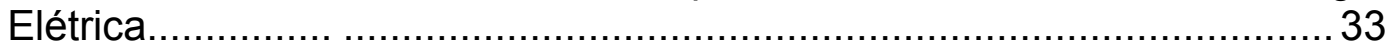

2.3. Manutenção Centrada na Confiabilidade ....................................... 36

2.3.1. A Manutenção, do Prelúdio à Evolução dos Dias Atuais ................. 36

2.3.2. Cronologia da MCC ........................................................... 40

2.3.3. Objetivos da Manutenção Centrada na Confiabilidade................... 43

2.3.4. Conseqüências de Falhas ...................................................... 44

2.3.5. Questões Básicas da MCC ...................................................... 45

2.3.6. Primeira Etapa - Seleção do Sistema e Coleta de Informações..... 46

2.3.7. Segunda Etapa - Análise de Modos de Falha, Causas e Efeitos ...47

2.3.8. Terceira Etapa - Seleção de Funções Significantes ........................ 59

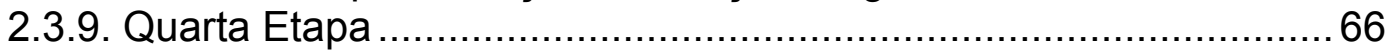

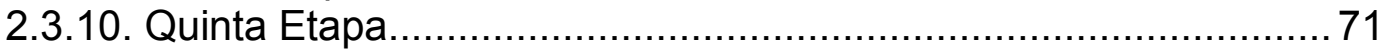

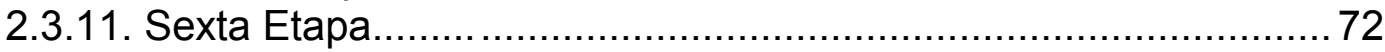

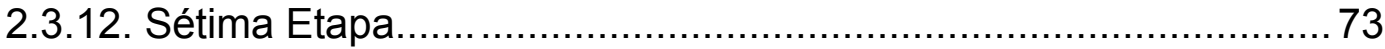

2.4. MCC no Setor Elétrico Brasileiro .................................................. 73

2.5. Projetos de Pesquisa em MCC …................................................. 75

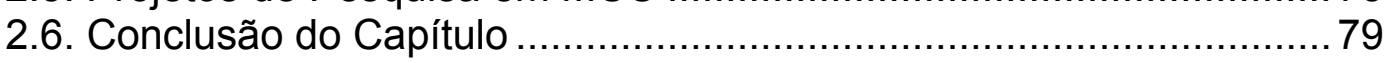

3. Avaliação da Confiabilidade de Sistemas de Proteção de Sistemas de Potência Através do Método da Freqüência e Duração ............................ 81

3.1. Conceitos do Processo de Markov .............................................. 82

3.1.1. Determinação das Probabilidades dos Estados em Função do

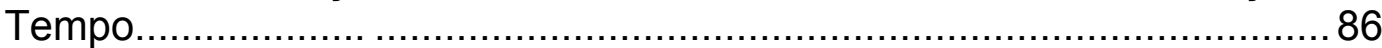

3.1.2. Probabilidade em Regime Permanente ..................................... 87

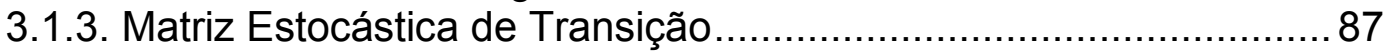


3.1.4. Determinação da Probabilidade dos Estados em Regime

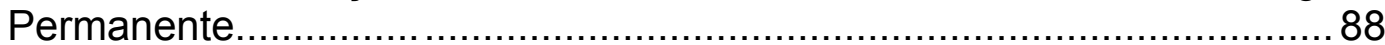

3.2. Método da Freqüência e Duração ................................................. 89

3.2.1. Conceitos de Freqüência e Duração ............................................ 89

3.3. Aplicação a Sistemas Multi-Estados .............................................. 92

3.4. Relação entre Intervalos de Manutenção Programada e a Confiabilidade de um Componente .................................................... 93 3.4.1. Relação do Tempo Médio Para Falha e o Aumento do Intervalo entre Manutenções Programadas .......................................................96 3.5. Processos de Decisão Markovianos em Sistemas de Segurança e Proteção [Siqueira, 1999] ................................................................. 97

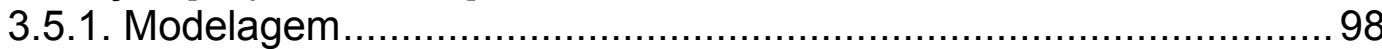

3.5.2. Agregação de Estados ........................................................... 99

3.5.3. Determinação da Taxa de Falha Interna do Sistema de

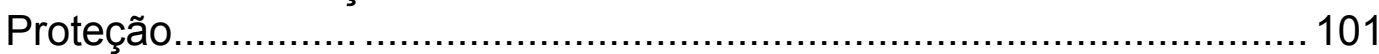
3.6. Determinação das Probabilidades em Regime Permanente dos Estados do Sistema de Proteção .................................................... 104

3.7. Conclusões e Comentários sobre o Modelo Adotado ...................... 105

\section{Modelo Markoviano para Equipamentos de Proteção de Sistemas}

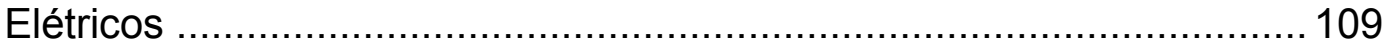

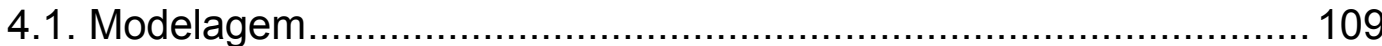

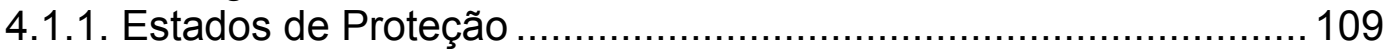

4.1.2. Diagrama de Estados do Sistema de Proteção........................... 110

4.1.3. Processos de Manutenção ......................................................... 113

4.1.4. Modelo de Estados Agrupados .............................................. 114

4.2. Análise do Modelo Markoviano …............................................. 120

4.2.1. Parametrização do Modelo..................................................... 120

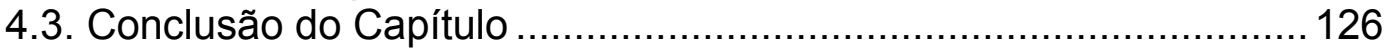

5. Modelo de Apoio à Decisão para Obtenção do Intervalo de Manutenção Programada do Sistema de Proteção de Linhas de Transmissão...........130 5.1. Análise de Sensibilidade dos Parâmetros do Modelo Markoviano em Função da Periodicidade da Manutenção Programada.........................130 5.1.1. Freqüência de Desligamentos Forçados dos Sistemas Protegidos Considerando Diferentes Intervalos Fixos de Manutenção

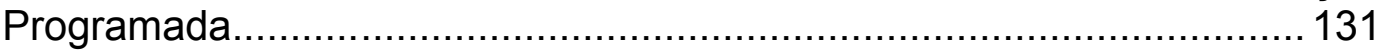
5.1.2. Freqüência de Falhas Devido a Recusas de Atuação da Proteção Considerando Diferentes Intervalos Fixos de Manutenção Programada.......................................................................... 135

5.2. Relação entre a Taxa de Falha e o Aumento do Intervalo Fixo de Manutenção Programada do Sistema de Proteção...............................136 5.3. Probabilidades em Regime Permanente dos Estados do Sistema de Proteção de Linhas de Transmissão em Função da Variação do Intervalo entre Manutenções....................................................................... 139 5.4. Cálculo do Desconto da Receita Anual Permitida Devido a Indisponibilidade do Sistema de Proteção ........................................... 143 5.4.1. Cálculo da Parcela Variável ...................................................... 144 
5.5. Desconto da RAP Devido a Indisponibilidades Programada e Não Programada em Função do Intervalo entre Manutenções ..................... 146 5.6. Determinação do Intervalo entre Manutenções Programadas em Função do Desconto da RAP por Indisponibilidades Programadas e Não Programadas.................................................................................. 148

5.6.1. Taxa Marginal de Substituição ............................................ 148 5.6.2. Determinação dos Intervalos de Manutenção Programada Através da Análise da Taxa Marginal de Substituição....................................... 151 5.6.3. Determinação da Curva "Risco x $\Delta R / \Delta P V "$ do Problema em Análise..........................................................................................155

5.6.4. Estabelecimento dos Níveis de Riscos Aceitáveis (Restrições do

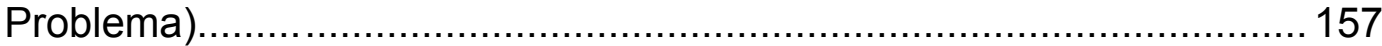

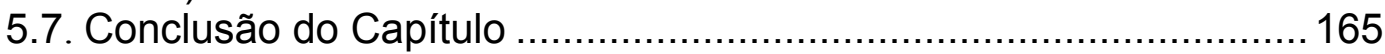

6. Conclusões e Trabalhos Futuros............................................... 167

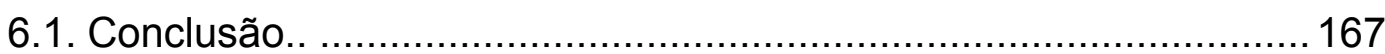

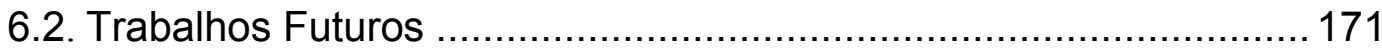

7. Referências bibliográficas .................................................. 173

Apêndice A - Processos de Decisão Markovianos em Sistemas de A.1. Modelagem ........................................................................ 181

A.1.1. Tipos de Manutenção........................................................ 181

A.1.2. Modos de Falha............................................................... 189

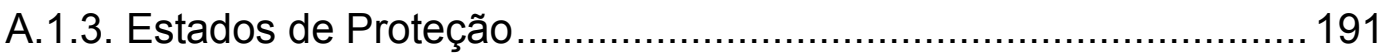

A.1.4. Sistema de Proteção............................................................ 192

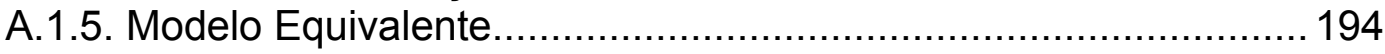

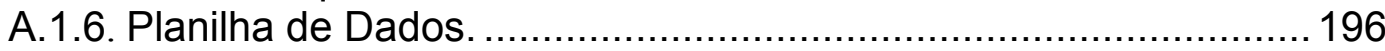

A.1.7. Processos de Manutenção ..................................................... 200

A.1.8. Processo Produtivo [Siqueira, 1999] ..................................... 203

Apêndice B - Teoria do Consumidor ............................................... 207

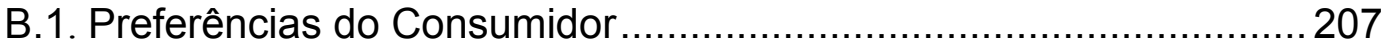

B.2. Curvas de Indiferença ........................................................... 208

B.3. Elasticidade-Preço da Demanda .............................................. 210

B.3.1. Elasticidade - Risco da Parcela Variável ................................ 211 


\section{Lista de Figuras}

Figura 1.1 - Cronologia dos Eventos da Cobrança da Parcela Variável.. 25

Figura 2.1 - Previsão para a Substituição de Equipamentos do Sistema de Distribuição de Energia da EDF........................................ 34

Figura 2.2 - Crescimento das Expectativas de Manutenção .................... 37

Figura 2.3 - Curva Idade vs Probabilidade de Falha ............................... 38

Figura 2.4 - Curva da Banheira .................................................. 39

Figura 2.5 - Padrões de Idade-Confiabilidade para Equipamentos Não Estruturais de Aeronaves...................................................... 41

Figura 2.6 - Diferentes Visões sobre Falhas..................................... 48

Figura 2.7 - Função Densidade de Falha Hipotética. $Q(t)$, Probabilidade do Equipamento Falhar Antes do Instante $t ; R(t)$, Probabilidade do Equipamento Sobreviver Após o

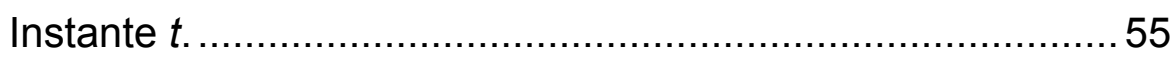

Figura 2.8 - Curva de Degradação da Resistência à Falha ...................... 70

Figura 2.9 - Critérios de Periodicidade na MCC ……........................... 75

Figura 3.1 - Ciclo de Vida de um Componente Qualquer ....................... 82

Figura 3.2 - Ciclo Operacional de Duas Unidades Transmissoras .......... 83

Figura 3.3 - Ciclo de Vida Médio de um Equipamento Qualquer .............. 85

Figura 3.4 - Diagrama de Espaço de Estados de um Equipamento Modelado a Dois Estados .................................................... 85

Figura 3.5 - A Função Densidade de Probabilidade do Ciclo de Vida de um Componente sob Manutenção Preventiva Periódica ....... 95

Figura 3.6 - (a) Relação do Tempo Médio para Falha e o Intervalo entre Manutenções; (b) Tempo Esperado para Falha Caso Não Seja Realizada Manutenção Programada ......................................97

Figura 3.7 - Diagrama de Transição de Estados do Sistema de

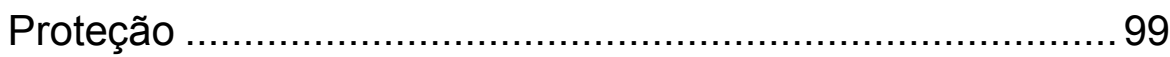

Figura 3.8 - Diagrama de Estados do Modelo Simplificado .................... 100

Figura 3.9 - Diagrama de Transição de Estados do Sistema de Proteção ........................................................................ 105

Figura 4.1 - Ciclo Operacional do Sistema de Proteção ......................... 111 
Figura 4.2 - Diagrama de Transições entre Estados 112

Figura 4.3 - Modelo Agrupado ......................................................... 115

Figura 4.4 - Diagrama de Transição de Estados (Agrupado) ................. 120

Figura 4.5 - Taxa de Falha dos Equipamentos de Proteção .................. 126

Figura 4.6 - Probabilidade de Permanência no Estado de Falha .......... 127

Figura 4.7 - Tempo Médio de Duração da Manutenção Programada do Sistema de Proteção ....................................................... 128

Figura 4.8 - Tempo Médio de Duração da Manutenção Corretiva do Sistema de Proteção ........................................................ 128

Figura 5.1 - Curva da Banheira Considerando-se um Intervalo Fixo de Manutenção Programada 131

Figura 5.2 - Variação da Taxa de Falha em Função do Aumento do Intervalo de Manutenção Programada 132

Figura 5.3 - Taxa de Falha em Função do Intervalo Fixo de Manutenções Programadas 133

Figura 5.4 - Taxa de Falha e Tempo Médio para Falha em Função da Variação do Intervalo Fixo de Manutenção Programada .... 137 Figura 5.5 - Probabilidade de Permanência no Estado NORMAL em Função da Variação do Intervalo Fixo entre Manutenções Programadas (Caso 1)...................................................... 140

Figura 5.6 - Probabilidade de Permanência nos Estados FALHA e TESTE em Função da Variação do Intervalo Fixo entre Manutenções Programadas (Caso 1) 141

Figura 5.7 - Probabilidade de Permanência no Estado NORMAL em Função da Variação do Intervalo Fixo entre Manutenções Programadas (Caso 2)................................................... 142

Figura 5.8 - Probabilidade de Permanência no Estado NORMAL em Função da Variação do Intervalo Fixo entre Manutenções Programadas (Caso 3).................................................. 142

Figura 5.9 - Curva de Indiferença do Consumidor (Risco vs

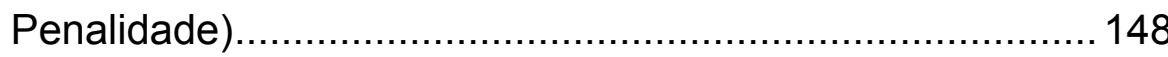

Figura 5.10 - Taxa Marginal de Substituição para uma Curva de Indiferença Contínua 149 
Figura 5.11 - Curva de Indiferença e Respectivas TMS ........................ 150

Figura 5.12 - Curva de Probabilidade de Falha x PV Programada........ 151

Figura 5.13 - Curva do Nível de Risco vs PV ...................................... 154

Figura 5.14 - Curva Característica "R vs $\Delta R / \Delta P V$ "............................... 154

Figura 5.15 - Variação da Probabilidade da Proteção Encontrar-se no Estado FALHA vs $\Delta R / \Delta P V$.............................................. 156

Figura 5.16 - Variação da Probabilidade da Proteção Encontrar-se no Estado FALHA vs $\Delta R / \Delta P V($ Caso 2).................................... 162

Figura 5.17 - Variação da Probabilidade da Proteção Encontrar-se no Estado FALHA vs $\triangle R / \Delta P V($ Caso 3).................................... 165

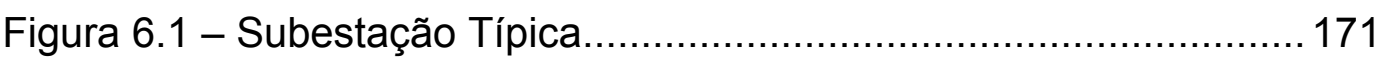

Figura A 1 - Lógica de Decisão ...................................................... 183

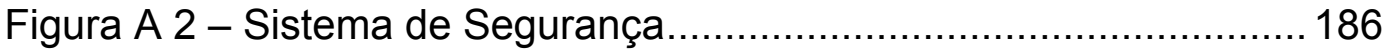

Figura A 3 - Transições entre Estados .............................................. 194

Figura A 4 - Modelo do Processo Produtivo ………............................. 204

Figura B 1 - Gráfico com os Tipos de Carnês....................................... 208

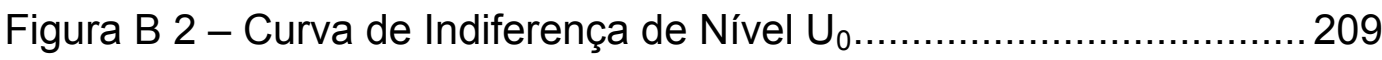

Figura B 3 - Mapa de Indiferença do Consumidor ................................ 210

Figura B 4 - Curva elasticidade - risco da PV ................................. 211 


\section{Lista de Tabelas}

Tabela 1.1 - Histórico da Contabilização da Parcela Variável no Brasil .. 26

Tabela 2.1 - Estrutura do Gerenciamento de Ativos ................................ 31

Tabela 2.2 - Modos de Falha Típicos de Componentes Elétricos ............51

Tabela 2.3 - Modelagem Matemática das Funções Densidades e

Distribuições de Probabilidade ..............................................57

Tabela 2.4 - Níveis de Detectabilidade de Risco ..................................... 61

Tabela 2.5 - Níveis de Freqüência de Modos de Falha ........................... 62

Tabela 2.6 - Níveis de Severidade de Risco............................................ 63

Tabela 2.7 - Níveis de Aceitabilidade de Risco ......................................... 64

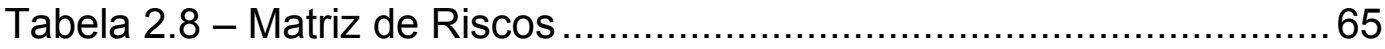

Tabela 2.9 - MCC no Setor Elétrico Brasileiro .......................................... 74

Tabela 2.10 - Projetos de Pesquisa em MCC..................................... 78

Tabela 4.1 - Tempos Médios para Teste e Reparo ............................. 114

Tabela 4.2 - Freqüência de Desligamentos Indevidos [ocorrências/

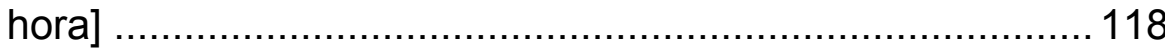

Tabela 4.3 - Freqüências de Recusas da Proteção [ocorrências/hora] 119

Tabela 4.4 - Taxa de Falha do Sistema de Proteção [falha/hora].......... 123

Tabela 4.5 - Probabilidades Permanentes dos Estados........................ 125

Tabela 5.1 - Freqüência de Falhas Devido a Desligamentos Indevidos do Sistema de Proteção de Linhas de Transmissão para Diferentes Intervalos Fixos de Manutenções Programadas (Caso 1) 134

Tabela 5.2 - Freqüência de Falhas Devido a Desligamentos Indevidos do Sistema de Proteção de Linhas de Transmissão (Caso 2).. 134

Tabela 5.3 - Freqüência de Falhas Devido a Desligamentos Indevidos do Sistema de Proteção de Linhas de Transmissão (Caso 3) .. 134

Tabela 5.4 - Freqüência de Falhas Causadas por Recusas de Atuação da

Proteção de Linhas de Transmissão (Caso 1)..................... 135

Tabela 5.5 - Freqüência de Falhas Causadas por Recusas de Atuação da Proteção de Linhas de Transmissão (Caso 2)......................136 
Tabela 5.6 - Freqüência de Falhas Causadas por Recusas de Atuação da Proteção de Linhas de Transmissão (Caso 3)..................... 136

Tabela 5.7 - Taxa de Falha e Tempo Médio para Falha em Função da Periodicidade da Manutenção 138

Tabela 5.8 - Probabilidades em Regime Permanente do Modelos de Estados em Função do Intervalo entre Manutenções (Caso 1) 139

Tabela 5.9 - Desconto da RAP por Indisponibilidade Programada em Função da Variação da Periodicidade da Manutenção (Caso 1) 147

Tabela 5.10 - Desconto da RAP do Sistema de Proteção de Linhas de Transmissão por Indisponibilidade Programada e a Variação da TMS (Caso 1) 152

Tabela 5.11 - "Risco vs $\Delta \mathrm{R} / \Delta \mathrm{PV}$ " (Caso 1). 155

Tabela 5.12 - Intervalos entre Manutenções Contidos na Fronteira de Eficiência de Risco 160

Tabela 5.13 - "Risco vs $\Delta \mathrm{R} / \Delta \mathrm{PV}$ " (Caso 2). 161

Tabela 5.14 - Intervalos entre Manutenções em Função dos Critérios (Caso 2) 163

Tabela 5.15 - Desconto da RAP do Sistema de Proteção de Linhas de Transmissão por Indisponibilidade Programada e a Variação da TMS (Caso 3) ........................................................ 163

Tabela A 1 - Manutenção Centrada na Confiabilidade ......................... 184

Tabela A 2 - Conseqüências de Falhas e Tipos de Manutenção .......... 188

Tabela A 3 - Modos de Falha de Sistema de Proteção ......................... 190

Tabela A 4 - Modelos e Sistemas Equivalentes ................................ 195

Tabela A 5 - Modelos Equivalentes .............................................. 196

Tabela A 6 - Dados das Instalações ................................................. 199

Tabela A 7 - Valores Esperados dos Tempos de Teste e de Reparo [h] ......................................................... 203

Tabela A 8 - Freqüência de Desligamentos Forçados $\left(F_{d}\right)[1 /$ hora].......206 Tabela B 1 - Tipos de Carnês Disponíveis ao Consumidor .................. 207 


\section{Lista de Abreviaturas e Siglas}

SIGLA UNIDADE

DESCRIÇÃO

A

ABNT

ANEEL

CA

CCT

CUST

CPST

DDP

DOD

EQP

$E[f]$

$f(t)$

$F_{i j}$

$F_{e} \quad f /$ hora

$F_{r} \quad f /$ hora

IF

IP

$\mathrm{K}_{\mathrm{p}}$

$\mathrm{K}_{\mathrm{o}}$

LA

MCC

$\mathrm{MC}$

$\mathrm{m}$

o

NP

NO
Disponibilidade

Associação Brasileira de Normas Técnicas

Agência Nacional de Energia Elétrica

Custo Anual

Contrato de Conexão ao Sistema de Transmissão

Contrato de Uso do Sistema de Transmissão

Contrato de Prestação de Serviço de Transmissão

Duração em minutos de cada desligamento programado que ocorra no mês

Duração em minutos de cada desligamento não programado que ocorra no mês

Números de equipamentos do sistema de proteção

Valor esperado para falha

densidade de falha

Freqüência de transição do estado i para o estado j

freqüências de desligamentos indevidos pela proteção

freqüências de recusa de atuação da proteção

Inspeção funcional

Inspeção preditiva

Fator para desligamentos programados. Normalmente igual a 10

Fator para outros desligamentos de até 300 minutos após o primeiro minuto (o fator será reduzido para $\mathrm{K}_{\circ} / 15$, após o $301^{\circ}$ minuto). Normalmente igual a 150

$\mathrm{R} \$ \quad$ Lucro Anual

Manutenção Centrada em Confiabilidade

Manutenção corretiva

Tempo médio de duração da manutenção programada

Tempo médio para falha

Número de desligamentos programados da instalação durante o mês

número de desligamentos não programados da instalação ao longo do mês 
$\mathrm{O}_{\mathrm{i}}$

ONS

$\mathrm{P}_{0}(\mathrm{t})$

$P_{1}(t)$

[P]

$\mathrm{P}_{\mathrm{i}} \quad \mathrm{pu}$

$P_{i j} \quad p u$

PB R\$

PV R\$

$Q(t)$

$\mathrm{R}(\mathrm{t})$

$r$

$\mathrm{r}_{\mathrm{i}}$

RA

RAP

RF

RP

SP

SO

$\mathrm{t}$

T

$t_{R}$

$U$

$\lambda(\mathrm{t})$

$\lambda$

$\lambda_{\mathrm{ij}}$

$\lambda_{f}$

$\mu$

$\mu_{\mathrm{ij}}$

$[\lambda]$

$\Omega$ tempo observado no estados "operando", para o i-ésimo ciclo Operador Nacional do Sistema Elétrico

probabilidade do equipamento estar operando no instante $t$ probabilidade do equipamento falhar no instante $t$

Matriz estocástica de transição entre estados

Probabilidade do estado $\mathrm{i}$

Probabilidade de transição do estado i para o estado j

Pagamento Base (mensal)

Parcela Variável da Receita Permitida

Função Distribuição de Falhas

Função de Sobrevivência

Tempo médio de duração de reparo de um equipamento

Tempo observado no estado "reparo" para o i-ésimo ciclo

Receita Anual

Receita Anual Permitida

Reparo funcional

Restauração preventiva

Substituição preventiva

Serviço operacional

hora Intervalo de tempo

hora Intervalo entre manutenções

hora Tempo de reparo

Indisponibilidade

Taxa de risco ou taxa de falha (Hazard Rate)

Taxa de transição de falha de um determinado equipamento

Taxa de falha entre os estados $i$ e $j$

f/hora Taxa de falha interna da proteção

Taxa de transição de reparo de um determinado equipamento

Taxa de reparo entre os estados $i$ e $j$

Matriz de transição

Fronteira de Eficiência de Risco 